\title{
The Analysis of The Efficacy and Safety of Transobturator Sling Operation by Using an Adjustable Sling Device in The Treatment of Stress Urinary Incontinence
}

\author{
Gokhan Temeltas ${ }^{1}$, Mehmet Bilgehan Yuksel ${ }^{1 *}$, Volkan Tatlı ${ }^{1}$, Bilal Gumus ${ }^{1}$
}

\author{
Article History: \\ Received September 2013 \\ Accepted October 2013 \\ Available online January 2014

\section{Keywords:} \\ Adjustable Sling Device \\ Stress Urinary Incontinence \\ Transobturator Sling \\ Corresponding Author: \\ Mehmet Bilgehan Yuksel, Celal \\ Bayar University, Faculty of \\ Medicine, Department of Urology, \\ Manisa Turkey. \\ Tel: +905056497081 \\ Email: \\ drmehmetvuksel@hotmail.com
}

\begin{abstract}
Objectives: We aimed to evaluate the safety and efficacy of a transobturator sling (TOT) procedure by using an adjustable TOT device in the treatment of stress urinary incontinence (SUI).

Materials and Methods: The data of 89 patients who had the diagnosis of SUI and underwent TOT operation by using an adjustable MUS device of SAFYRE t plus (Promedon $\AA$ ) between June 2005 - November 2012, were retrospectively evaluated. The pateints were evaluted by the parametres of physical examination, stress test, ultrasonography, uroflowmetry, residual urine measurement, cystometry, subjective incontinence scoring (VASi), patients' satisfaction scoring (VASs), and ICQ-SF questionare. The preoperative, perioperative, and postoperative collected data were statistically analysed to determine the efficacy and safety of TOT application by using SAFYRE t plus.

Results: The mean age, account of parity, and body mass index was $55.3 \pm 10.1,2.34 \pm 1.43$, and $24.97 \pm 3.7$, respectively. While the pre- and postoperative results of stress test, VASi, and ICIQ-SF scores were significantly different $(\mathrm{P}=0.00$ in all), the pre- and postoperative results of Qmax and PVR account were similar $(\mathrm{P}=0.84, \mathrm{P}=0.79$, respectively). The severity of subjective incontinence (VASi score) significantly improved after the operation. The mean VASs score at postoperative 12th month was $7.75 \pm 1.11$. The subjective and objective cure rates were $\% 89.9$ (80/89) and \%78.6 (70/89), respectively.

Conclusion: The TOT operation by using SAFYRE t plus, which was an adjustable sling device, seemed to be an efficient, reasonably safe, minimally invasive treatment alternative for the surgical management SUI in women.
\end{abstract}

1- Celal Bayar University, Faculty of Medicine, Department of Urology, Manisa, Turkey. 


\section{Introduction:}

In a healthy woman, the pubocervical fascia, which is an important structure of the pelvic anatomy, constructs a suburethral hammock that supports the urethra. During some actions, such as coughing, the increase in abdominal pressure causes the prolapsus of bladder neck and urethral compression that result in urine leakage, which is defined as stress urinary incontinence (SUI). A normal anatomy and function of the pubocervical fascia supports the urethra and bladder neck and avoids the occurrence of SUI. An incompetence in this support results in the occurrence of SUI. Thus, the major disorder in the occurrence of SUI is accepted as a structural defect in the pelvic structure supporting the normal functional anatomy of lower urinary system. Therefore, the surgical treatment modalities take the greatest place in the management of SUI (1, 2).

There have been many attempts to develop surgical techniques and products that would result in the long-term successful treatment of SUI. Techniques have included vaginal, open abdominal, laparoscopic, and slingtype procedures, with the goal of achieving balance in terms of efficacy, safety, and simplicity. Midurethral sling (MUS) procedures have been the mainstay in treatment of SUI over the last decade. The surgical management of SUI related to urethral hypermobility has been significantly changed since the development of retropubic placement of suburethral tension-free vaginal tape (TVT) that was first described by Ulmsten and Petros in 1995 (3). TVT procedure was a prototype, minimally invasive intravaginal suburethral sling operation and accepted worldwide as the standard surgical treatment for SUI for along time $(4,5)$. Although TVT is an effective treatment with the success rates ranging from $81 \%$ to $95 \%$ (6- 9), a wide variety of the complications including visceral (bowel, bladder, urethra), vessel (iliac vessels), and nerve injuries have been described. Many of these complications seem to be related to the blind upward vaginal passage of the metallic needles through the retropubic space $(5,10-12)$. In an attempt to minimize the complications of TVT, a new MUS called transobturator tape (TOT) was created by Delorme in 2001, where the needle passers run the sling through the obturator foramen (13). TOT procedure significantly decreased the complications of TVT with an equivalent cure rate (13). Some metaanalyses in the literature showed that no significant difference was determined between the success rates of TVT and TOT (14-16).

The aim of this study is to evaluate the safety and efficacy of the transobturator suburethral sling procedure by using an adjustable transobturator suburethral sling device (SAFYRE) in the treatment of SUI and to present the experience of a single urological center in this area.

\section{Material \& Methods:}

The data of 89 patients who had the diagnosis of SUI and underwent TOT operation by using an adjustable MUS device, which was called SAFYRE $t$ plus (Promedon $\AA$ ) between June 2005 November 2012, were retrospectively evaluated. The patients who were with the diagnosis of pure stress incontinence, had a TOT surgery by using only SAFYRE, and had no other disorders at urodynamic testing were included in the study. A hystory of previous major pelvic surgiries, such as major gynecologic operations, urodynamically proved detrusor overactivity, postvoid residual urine measurement (PVR) $>100 \mathrm{ml}$, the presence of urinary infection, negative cotton swab test (Q-type test less than $30^{\circ}$ ) were the exclusion criterias. Previous to the surgery, all patients were assessed with a detailed medical history, physical examination, stress test (cough test), urinary ultrasonography, uroflowmetry, (maximal flow rate $=$ Qmax), PVR measurement, cystometry, subjective incontinence scoring (VASi) that is a single, self-answered, Likert-type scale of 0 - 10 that grades the the severity of incontinence: 0 represents "no incontinence" and 10 represent "severe incontinence", and ICQ-SF questionares. All data were recorded in our database.

Sling device description : 
The SAFYRE t plus adjustable sling system is the latest step in the development of TOT procedures and combines the safety of TOT procedure and an optimized adjustability. It is designed for being positioned through the medial aspect of the obturator membrane, underneath the ischiopubic ramus bilaterally, thus it provides midurethral support. However, the developed system of SAFYRE $t$ plus provides a controlled tensioning according to the patient requirement. The surgical kit consists of a macroporous monofilament size polypropylene mesh, two anatomically designed needles for each hand, and two rings. The rings that help the stabilization of desired tension of the urethra, if it is required, keep the desired tension postoperatively. The anatomically designed needles are with a unique semisircular design simplifiying the surgical movement and the transobturator passage. Furthermore, the differential diameter betwen 3.25- $4 \mathrm{~mm}$ facilitates easy handling of the needles and the maneuverability during the transport of the mesh. The mesh is made of a macroporous monofilament polypropylene material as a suburethral hammock supporting the urethra and connected to two fixation arms that is made from solid polydimethylsiloxane elastomer. The fixation arms are made up of a multiple cone structure.

Surgical procedure :

All patients were preoperatively informed about the procedure. At the operation day, a second generation of cephalosporine was applied to provide infection prophylaxia (Cephazoline $1 \mathrm{gr}$, IV infusion). The cases were prepared in lithotomy position under spinal anesthesia. The surgical area was cleaned, and a foley catheter was inserted in. TOT operation was performed by using only SAFYRE t plus sling device. A 2-cm lenght vertical incision was performed on the anterior vaginal mucosa, at $1 \mathrm{~cm}$ proximally from the urethral meatus, along the midurethra. The area below the fascia was minimally dissected laterally towards the ascending ramus of the ischiopubic bone, preserving the endopelvic fascia bilaterally. A $1 \mathrm{~cm}$ lenght skin incision was performed on the vanishing point of the genitofemoral fold with the level of clitoris. Subsequently, the operator's left index finger was inserted into the vaginal incision on the patients' left side. The tip of right-handed SAFYRE t plus needle was pushed forward through the obturator membrane. After the filling of a pop reflecting the perforation of the obturator membrane, the needle was rotated approximately $45^{\circ}$ along the posterior surface of the ischiopubic ramus towards the left index finger, and the tip was taken out of the vaginal incision by pushing of the needle in the guidance of the left index finger. After the determination of the tip of needle, the fixation arm of the mesh with a solid, multiple cone structure was attached to the tip of the needle and pulled out through the skin incision in the genitofemoral sulcus. All steps of the same procedure was applied for the right side of the patient. Subsequently, it was determined that the mesh was in appropriate and flat position covering the midurethra, and the appropriate tension was adjusted by the placement of a surgical clemp between the urethra and mesh. Subsequently, the rings were attached to the fixation arms and placed into the incision in genitofemoral sulcus. The spare parts of the fixation arms were truncated above the rings, and the rings and tips of the fixation arms were covered with the closure of the skin incision by suturing over them. After the achievement of appropriate hemostasis and the control of any perforation inside the vaginal wall related to the needle passage, the vaginal incision was also closed over the mesh. A cystoscopical examination was also performed in all subjects at the end of the procedure.

At postoperative period, foley catheter was removed at postoperative first day. Subsequently, when the urination was realized, the patient was discharged at the same day. The cases of not having any urine leakage at stress test or no pathological findings at urodynamic testing were accepted as the success of the procedure.

At the follow up period, the analyses of physical examination, stress test (cough test), urinary ultrasonography, uroflowmetry ( Qmax), PVR measurement, cystometry, subjective incontinence scoring (VASi), patients' satisfaction scoring (VASs) 
that is a single, self-answered, Likert-type scale of $0-10$ that grades the degree of patients' satisfaction regarding continence: 0 represents "not satisfied" and 10 "satisfied", and ICQ-SF questionares were also executed at postoperative 12th month. All data were also collected. While objective cure was defined as the absence of urine leakage during the stress test, subjective cure was accepted as a VASs score $\geq 7$. The preoperative, perioperative, and postoperative data were statistically analysed to determine the efficacy and safety of the TOT application by using SAFYRE $t$ plus.

Statistical analyses were performed by using SPSS version 18.0, and the data were displayed as mean \pm standard deviation (SD) (range). Continous variables were evaluated with Paired Student's t test or Wilkoxon signed-rank test. Categorical variables were evaluated with the ChiSquare tests. A 5\% level of significance was used for all statistical testing. A $p$ value $<0.05$ was considered significant.

\section{Results:}

89 patients were diagnosed as SUI and underwent a TOT operation by using only the MUS device of SAFYRE $t$ plus were included in the study. The mean age was found $55.3 \pm 10.1$ years. The mean account of parity was $2.34 \pm 1.43$. The mean body mass index was determined as $24.97 \pm 3.7$. In our sample, while postmenaposal women constite \%38.2 (34/89) of the patients, $\% 16.9(15 / 89)$ of patients were with the prolapsus. The pre- and postoperative stress tests was positive in 85 (\%95.5) and 14 (\%15.7) patients, respectively. The mean pre- and postoperative values of VASi score and maximal flow rate (Qmax) were $7.56 \pm 2.03$ and $2.24 \pm 1.11$, and $23.26 \pm 5.36$ and $23.01 \pm 5.52 \mathrm{ml} / \mathrm{dk}$, respectively. The mean value of pre- and postoperative PVR value and ICIQ-SF score was found $6.12 \pm 5.62$ and $6.49 \pm 5.59$, and $14.07 \pm 3.08$ and 5.76 \pm 3.02 . The comparison of pre- and postoperative values of these parametres revealed that while there were significant differences between the pre- and postoperative values of stress test, VASi, and ICIQ-SF scores ( $\mathrm{P}=0.00$ in all), the pre- and postoperative results of Qmax and PVR account were similar $(\mathrm{P}=0.84, \mathrm{P}=0.79$, respectively). These results revealed that the severity of subjective incontinence (VASi) significantly improved after the TOT operation by using SAFYRE $t$ plus MUS device. These data were clearly seen in Table1 and Figure1. In addition, the mean VASs score at postoperative 12th month, which reflected the patient satisfaction regarding the continence after the operation, was found as $7.75 \pm 1.11$. This was a positive result for subjective cure. The analysis of the cure rates of SAFYRE $t$ plus MUS application showed that subjective cure was obtained in the rate of \%89.9 (80/89). However, the objective cure rate was found \%78.6 (70/89).

\section{Discussion:}

Urinary incontinence (UI) was described by International Continence Society as a condition where involuntary loss of urine is a social or hygienic problem and is objectively demonstrable (17). While the most common type of UI in women is SUI, it is defined as the leakage of urine as a result of some actions, such as coughing, sneezing, or physical activities, that increase intraabdominal pressure without any detrusor contractions (1). Suburethral hammock theory regarding the structural support of the urethra was described by DeLancey, and it is accepted that the loss of this support is commonly related with urethral hypermobility. Urethral hypermobility is a common factor that is commonly seen in most of SUI cases (18). Although pelvic floor muscle exercise is the first choice of treatment for SUI and is known to be effective in approximately $\% 50$ of cases, surgical treatment alternatives is indicated when conservative treatment fails or the patient wants definitive treatment (19). In the surgical treatment modalities, MUS procedures, which was the minimally invasive operations for the treatment of female SUI, have been the mainstay in treatment of SUI over the last decade.

Initially, Ulmsten et al. defined TVT operation, which was a prototype, minimally invasive intravaginal suburethral sling operation and accepted worldwide as the standard surgical treatment for SUI $(4,5)$. The previous studies showed that TVT is an 
effective and safe MUS procedure with the cure rates between \%81- 95 (6- 9). The simple applicability, the applicability also under local anesthesia, minimally invasive character, and high cure rates increasingly popularized the use of TVT. However, a wide variety of the complications including visceral (bowel, bladder, urethra), vessel (iliac vessels), and nerve injuries are commonly seen in TVT procedure as a result of blind upward trocar passage of TVT device from vagina through the retropubic space $(5,10-12)$. Most of the researchers tried to minimize the severity and frequency of these complications. As a result of this effort, a new MUS technique was described by Delorme in 2001, where the needle passers run the sling through the obturator foramen (13). In this technique, a specially designed helical needle is used for the mesh placement by transobturator approach, and this mesh mimicks the structure and function of the pubocervical fascia. The retropubic passage of the needle is avoided, and a V-shaped suburtehral hammock of support is provided for the urethra instead of urethral suspension (13). Thus, TOT procedure can significantly decrease TVT related complications and also maintain or improve the cure rate of TVT procedure (13). In the previous studies, it was reported that TOT operation was an effective and safe procedure in the treatment of SUI with an $83 \%$ cure rate and a 5.4\% improvement at 6 months follow-up $(13,20)$. However, some studies comparing TOT with TVT revealed that although no significant difference was determined between the success rates of TVT and TOT, TOT was found the more easy and safe procedure (14-16). In our study, it was found that the mean subjective and objective cure rates after the operation were $\% 89.9$ and $\% 78.6$, respectively. These result were in accordance with the previous literature and showed that SAFYRE $t$ plus MUS application was an effective and minimally invasive treatment alternative for the management of SUI. When we looked at the complications of TOT operation, one study declared that TOT operation significantly decreased the total complication rate, which can increase up to $15 \%$ in TVT procedure, to the rate of $\% 2.2$
$(21,22)$. Urine retention, voiding difficulty, and de novo urgency were additional complication related to TVT operation. It was shown that while voiding difficulty and/or urine retention was with the rate of $\% 8-17$, de novo urgency had the rate of \%515 in TVT operation (23-26). Nevertheless, it has been reported that no voiding difficulty was determined in the previous study of Delorme using the TOT procedure (13). The comparison of urinary retention rates between TVT and TOT showed the rates of $\% 25$ versus $\% 13$, respectively. Furthermore, the rate of de novo urgency was reported as \%6.5 in TVT and \%6.7 in TOT, and this results was similar (27). Organ and vessel injuries including bladder, bowel perforations, iliac vessel imjuries can particularly occur during the blind trocar passage of TVT thorough the retropubic area. While it was reported that these complications could be seen in TVT procedure with the rate $\% 2-4$, no significant hemorragical complication has been reported in TOT operation. It was accepted that TOT procedure could minimize the occurrence of this kind of complications (28, 29). It was seen that no major complication related to the TOT operation, such as bladder perforation, bowel perforation, urethral perforation, vessel injury, and obturator hematoma, occurred in our study. Two patients (\%2.2) reported postoperative voiding difficulty that was required division of the tape 3 months after the surgery. In 1 patient (\%1.1), it was determined that a vaginal erosion occurred 10 months after the operation. The mesh was situated on the midline of midureathra, and it was treated by the excision of a large part of the mesh. In addition, two patients (\%2.2) presented groin pain, and these patients were managed by conservative treatment. Nevertheless, no cases of vaginal erosion were found in our sample at the follow-up visit. The results of our study regarding the complications were similar to the previous literature and showed that SAFYRE t plus MUS application was a safe and minimally invasive treatment alternative for the management of SUI.

\section{Conclusion :}

It was supposed that TOT surgery by using SAFYRE t plus MUS device was an efficient, 
reasonably safe, minimally invasive treatment alternative for the surgical management SUI in women. The advantages of simple application process, short duration of surgery and hospital stay, decreased healing time, minor postsurgical discomfort, and a limited number of complications seems to be made this method acceptable for the patients with SUI.

\section{Conflict of interest statement:}

We declare that we have no conflict of interest.

\section{Acknowledgments:}

We have no acknowledgements to disclose. The authors declared that they had no financial conflicts of interest or other interests that may influence the manuscript.

Table 1. The demonstration of preoperative and postoperative values of the parameters with the mean and standard deviation (SD) values.

\begin{tabular}{lccc}
\hline & $\begin{array}{c}\text { Preoperative value } \\
(\text { Mean } \pm \text { SD) }\end{array}$ & $\begin{array}{c}\text { Postoperative value } \\
(\text { Mean } \pm \text { SD) }\end{array}$ & P value \\
\hline Positive stress test & $85(\% 95.5)$ & $14(\% 15.7)$ & 0.00 \\
Subjective incontinence score (VASi) & $7.56 \pm 2.03$ & $2.24 \pm 1.11$ & 0.00 \\
Maximal flow rate & $23.26 \pm 5.36$ & $23.01 \pm 5.52$ & 0.84 \\
PVR measurement & $6.12 \pm 5.62$ & $6.49 \pm 5.59$ & 0.79 \\
ICIQ-SF score & $14.07 \pm 3.08$ & $5.76 \pm 3.02$ & 0.00 \\
\hline
\end{tabular}

Figure1. Preoperative and postoperative values of the Positive stress test, Subjective incontinence score (VASi), Maximal flow rate, PVR measurement and ICIQ-SF scores of stress incontinence patients. $\left(\mathrm{n}=89\right.$, Mean \pm Standard Deviation (SD), ${ }^{*} \mathrm{P}<0.001$

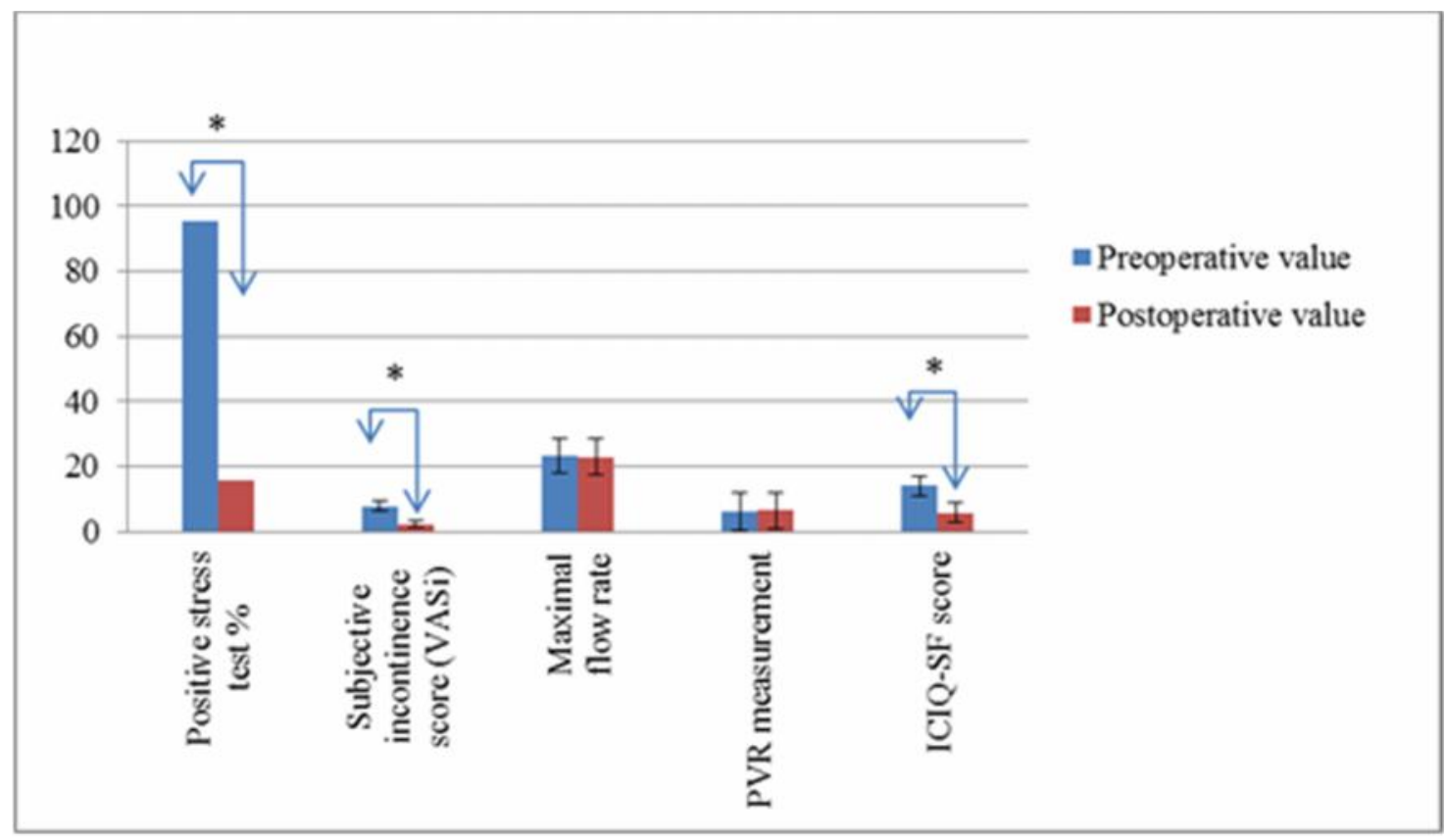




\section{References:}

1. Abrams P, Cardozo L, Fall M, Griffiths D, Rosier P, Ulmsten U, et al. Standard- isation Subcommittee of the International Continence Society. The standardisation of terminology of lower urinary tract dysfunction: report from the Standardization Sub-committee of the International Continence Society. Neurourol Urodyn. 2002; 21:167-178.

2. Petros PE, Ulmsten UI. An integral theory of female urinary incontinence. Experimental and clinical considerations. Acta Obstet Gynecol Scand Suppl 1990; 153:7-31.

3. Ulmsten U, Petros P. Intravaginal slingplasty (IVS): an ambulatory surgical procedure for treatment of female urinary incontinence. Scand J Urol Nephrol 1995; 29:75- 82.

4. Fong ED, Nitti VW. Review article: Mid-urethral synthetic slings for female stress urinary incontinence. BJU Int 2010; 106:596-608.

5. Ulmsten U, Henriksson L, Johnson P, Varhos G. An ambulatory surgical procedure under local anesthesia for treatment of female urinary incontinence. Int Urogynecol J Pelvic Floor Dysfunct. 1996; 7:81-6.

6.Ulmsten U, Johnson P, Rezapour M. A three-year follow up of tension free vaginal tape for surgical treatment of female stress urinary incontinence. Br J Obstet Gynaecol 1999; 106:34550 .

7.Nilsson CG, Kuuva N. The tension free vaginal tape procedure is successful in the majority of women with indications for surgical treatment of urinary stress incontinence. Br J Obstet Gynaecol 2001; 108:414-9.

8. Debodinance P, Delporte P, Engrand JB, Boulogne M. Tension-free vaginal tape (TVT) in the treatment of urinary stress incontinence: 3 years experience involving 256 operations. Eur J Obstet Gynecol Reprod Biol 2002; 105:49-58.

9.Meschia M, Pifarotti P, Bernasconi F, Guercio E, Maffiolini M, Magatti F, et al. Tensionfree vaginal tape: analysis of outcomes and complication in 404 stress incontinence women. Int Urogynecol J 2001; 12:24-7.

10. Ward KL, Hilton P, Browning J. A randomized trial of colposuspension and tension free vaginal tape for primary genuine stress incontinence. BJOG 2008;115:226-33.

11. Nilsson CG, Palva K, Rezapour M, Falconer C. Eleven years prospective follow-up of TVT procedure for treatment of stress urinary incontinence. Int Urogynecol J 2008; 19:1043-7.

12. Petros PE, Richardson PA. Midurethral Tissue Fixation System sling -- a 'micromethod' for cure of stress incontinence -- preliminary report. Aust NZJ Obstet Gynaecol. 2005; 45:3725 .

13. Delorme E. Transobturator urethral suspension: mini-invasive procedure in the treatment of stress urinary incontinence in women. Prog Urol 2001; 11:1306-13. 
14. Sung VW, Schleinitz MD, Rardin CR, Ward RM, Myers DL. Comparison of retropubic vs transobturator approach to midurethral slings: a systematic review and meta-analysis. Am J Obstet Gynecol 2007; 197:3-11.

15. Latthe P, Foon R, Toozs-Hobson P. Transobturator and retropubic tape procedures in stress urinary incontinence: a systematic review and meta-analysis of effectiveness and complications. BJOG 2007; 114:522-31.

16. Novara G, Artibani W, Barber MD, Chapple CR, Costantini E, Ficarra V, et al. Updated systematic review and meta-analysis of the comparative data on colposuspension, pubovaginal slings, and midurethral tapes in the surgical treatment of female stress urinary incontinence. Eur Urol 2010; 58: 218-38.

17. Bates P, Bradley WE, Glen E, Griffiths D, Melchior H, Rowan D, et al. The standardization of terminology of lower urinary tract function. J Urol 1979;121:551-4.

18. DeLancey JO. Structural support of the urethra as it relates urinary incontinence: the hammock hypothesis. Am J Obstet Gynaecol 1994; 170:1713- 20.

19.Bo K, Talseth T, Holme I. Single blind, randomized controlled trial of pelvic floor exercises, electrical stimulation, vaginal cones and no treatment in management of genuine stress incontinence in women. BMJ 1999;3 18:487-503.

20. Delorme E, Droupy S, de Tayrac R, Delmas V. Transobturator tape (Uratape): A new minimally-invasive procedure to treat female urinary incontinence. Eur Urol 2004;45:203- 7.

21. Costa P, Grise P, Droupy S, Monneins F, Assenmacher C, Ballanger P, et al. Surgical treatment of female stress urinary incontinence with a trans-obturator-tape (TOT) uratape: short term results of a prospective multicentric study. Eur Urol 2004; 46:102-7.

22.Lebret T, Lugagne PM, Herve JM, Barre P, Orsoni JL, Yonneau L, et al. Evaluation of tension-free vaginal tape procedure. Its safety and efficacy in the treatment of female stress urinary incontinence during the learning phase. Eur Urol 2001; 40:543-7.

23. Ulmsten U. The basic understanding and clinical results of tension-free vaginal tape for stress urinary incontinence. Urologe A 2001; 40: 269-73.

24. Soulie M, Cuvillier X, Benaissa A, Mouly P, Larroque JM, Bernstein J, et al. The tensionfree transvaginal tape procedure in the treatment of female urinary stress incontinence: a French prospective multicentre study. Eur Urol 2001; 39:709-14.

25. Arunkalaivanan AS, Barrington JW. Randomized trial of porcine dermal sling (Pelvicol implant) vs. tension-free vaginal tape (TVT) in the surgical treatment of stress incontinence: a questionnaire-based study. Int Urogynecol J Pelvic Floor Dysfunct 2003; 14:17-23.

26. Boustead GB. The tension-free vaginal tape for treating female stress urinary incontinence. BJU Int 2002; 89:687-93. 
27.De Tayrac R, Deffieux X, Droupy S, Chauveaud-Lambling A, Calvanese- Benamour L, Fernandez H. A prospective randomized trial comparing tension-free vaginal tape and transobturator suburethral tape for surgical treatment of stress urinary incontinence. Am J Obstet Gynecol 2004;190: 602-8.

28. Richards SR, Balaloski SP. Vulvar hematoma following a transobturator sling (TVT-O). Int Urogynecol J 2005; 23:1-2.

29.Wang AC, Lin YH, Tseng LH, Chih SY, Lee CJ. Prospective randomized comparison of transobturator suburethral sling (Monarc) vs suprapubic arc (SPARC) sling procedures for female urodynamic stress incontinence. Int Urogynecol J 2006; 17:439-43. 